\title{
Bronchial Artery to Pulmonary Artery Fistula Presenting with Massive Hemoptysis in a Pediatric Patient
}

\author{
Aoife Corcoran ${ }^{1}$ and Silvia Cardenas ${ }^{2}$ \\ ${ }^{1}$ Cleveland Clinic \\ ${ }^{2}$ Cleveland Clinic Children's Hospital
}

July 13, 2021

\begin{abstract}
Hemoptysis is a serious and potentially life-threatening event. Mortality is estimated at $13 \%$ for this chief complaint with age, volume of hemoptysis and receipt of blood products as risk factors for mortality. Hemoptysis is mostly seen in those with underlying congenital cardiac conditions or Cystic Fibrosis. We describe a unique case of a previously healthy 10 year old male who presented to the ED by EMS with a moderate volume episode of hemoptysis. He was admitted to the PICU where a sudden episode of massive hemoptysis precipitated by forced respiratory effort occurred during his examination. He decompensated and was emergently brought to the OR for airway evaluation by ENT and pulmonology. A large clot was found in the RML segment with brisk bleeding following removal of the clot. A 5 Fr bronchial blocker was placed to achieve hemostasis. Bronchial artery angiogram by IR demonstrated extravasation of contrast from right bronchial artery to segmental right lower lobe pulmonary artery shunt. He underwent embolization of the right bronchial artery. He was extubated the following day after no recurrent bleeding was confirmed with bronchoscopy. BA-PA fistulas are rare vascular anomalies in which an anastomosis is formed between systemic and pulmonary arteries. They are most commonly acquired, often described as secondary to chronic inflammatory lung diseases. BA-PA fistulas can also be congenital and have been seldom described in the literature. Our case highlights the importance of this rare diagnosis, which must remain on a pediatric pulmonologist's differential due to the significant associated mortality.
\end{abstract}

Bronchial Artery to Pulmonary Artery Fistula Presenting with Massive Hemoptysis in a Pediatric Patient

Brief Title - Massive Hemoptysis in a Pediatric Patient

Aoife Corcoran, MDa

Silvia Cardenas, $\mathrm{MD}^{\mathrm{b}}$

${ }^{a}$ Department of Pediatrics, Cleveland Clinic Children's, Cleveland, Ohio

${ }^{\mathrm{b}}$ Center for Pediatric Pulmonary Medicine, Cleveland Clinic Children's, Cleveland, Ohio.

\section{*Corresponding Author}

Silvia Cardenas, MD

Center for Pediatric Pulmonary Medicine, Cleveland Clinic Children's,

R3 9500 Euclid Avenue, Cleveland, OH 44195

Ph. 216.444.8453 Fax. 216.442.5185

CARDENS@ccf.org 


\section{Key Words:}

Hemoptysis

Pulmonary Vascular Disorders

Congenital Malformations

Bronchial artery embolization

Interventional pulmonology

Interventional radiology

Bronchoscopy

Source of funding : Not applicable Conflict of interest: None

Prior presentation : Poster presentation at ATS 2021

Introduction

Hemoptysis or expectoration of blood from the airways is a serious and often life-threatening event that is, thankfully, rare in pediatric patients. Mortality associated with hemoptysis is caused by asphyxiation not exsanguination. Children tend to swallow their sputum and thus, hemoptysis may go initially un-noticed (Pianosi P, Al-sadoon H 1996).

The etiology of hemoptysis in pediatric patients is numerous, ranging from the common, infection to the rare, vasculitides (Shnayder $\mathrm{R}$ et al, 2018). The clear majority of hemoptysis occurs in patients with underlying pulmonary and/or cardiac disease states and is rare in previously healthy children. Hemoptysis secondary to vascular anomalies in pediatric patients has been detailed in the past 20 years, however it has been largely attributed to arteriovenous malformations (AVMs) (Villardo RJM et al, 2011). Bronchial artery to pulmonary artery (BA-PA) fistula is an exceedingly rare condition seen in children.

\section{Case Description}

A previously healthy 10-year-old boy presented to our pediatric emergency department (ED) with complaints of large volume hemoptysis one hour prior to arrival in the setting of scant hemoptysis for the past 3 days. He reported initially coughing up small amounts of blood-tinged sputum that steadily increased in volume over the preceding 72 hours. Coughing was accompanied by mild left-sided chest wall pain but no pleuritic chest pain. Our patient reported being otherwise well over the past 3 days with no fever, nasal congestion, headaches or GI symptoms noted.

Upon arrival in the ED, he was awake, alert and in no acute distress with heart rate of 90 , blood pressure of $114 / 64$, oral temperature of 98.4 , respiration rate of 18 and $\mathrm{SpO} 2$ of $97 \%$ on room air. He was noted to have dried blood in his mouth and nose. No other focal findings noted on initial exam, with clear air entry bilaterally noted on respiratory exam. Laboratory work did not reveal any evidence of anemia (haemoglobin of 14.7), electrolyte or liver function abnormalities, hematuria, infection or coagulopathy. Chest x-ray demonstrated patchy consolidation in the right lower lobe which was further characterised on CT chest as abnormal patchy ground-glass opacities in the right middle and lower lobes suggestive of pulmonary haemorrhage (figure 1a).

Upon arrival to our PICU, our patient experienced another episode of hemoptysis of with desaturation to $86 \%$ which resolved with 2 litres of oxygen $\mathrm{FiO} 2$ 100\% via nasal cannula (NC). He remained stable on 2L NC overnight. The following morning during examination, 2 episodes of hemoptysis occurred in quick succession with desaturation to the low 80s, tachypnea to the 30s with diminished air entry in the right mid and lower zones with late inspiratory crackles. This prompted emergent transfer to the operating room for airway evaluation by pediatric otolaryngology, pediatric pulmonology and adult interventional pulmonology. 
Flexible nasal endoscopy and microlaryngoscopy revealed no bleeding in the nasal cavity, nasopharynx, oral cavity, supra-glottis, glottis or sub-glottis but did note streaks of blood in the trachea with no obvious bleeding source in the trachea. Flexible bronchoscopy revealed the normal anatomy and normal mucosa of the left lung to the first sub-segmental level. Evaluation of the tracheobronchial tree in the right lung revealed scant fresh blood and small blood clots over the trachea and right mainstem bronchus. A large blot clot that was partially obstructing the airway was found in the right middle lobe (RML) and in the right lower lobe (RLL) (figure 1b). Upon removal of the clot, brisk hemorrhage occurred from the RML. A Fogarty balloon (\#4) was placed in the RML by interventional pulmonology until hemostasis was achieved. A 5F Arndt ${ }^{\mathrm{TM}}$ bronchial blocker was placed, driven to the RML and inflated to occlude this bronchus.

Interventional radiology were called to the OR for emergent angiography and embolization. Thoracic aortogram via the right common femoral artery demonstrated a hairpin-like artery corresponding to Adamkiewicz artery supplying the right mainstem bronchus. Angiogram of the Adamkiewicz artery revealed a fistula between the right bronchial artery and segmental pulmonary artery to the right lower lobe (figure 1c). The right bronchial artery was embolized with final angiogram demonstrating patency of the proximal Adamkiewicz artery with occlusion of the distal vessel including right bronchial artery and bronchial to pulmonary artery fistula (figure 1d). A right pulmonary artery angiogram was performed post embolization and demonstrated no thrombosis, AVM, aneurysm or pseudoaneurysm (figure 1e). Our patient remained intubated with bronchial blocker in place for a further 24 hours with repeat bronchoscopy on post-op day (POD) 1 showing no evidence of further bleeding. Patient was extubated following removal of the bronchial blocker on POD 1. Full evaluation for bleeding diathesis and echocardiogram found no additional cause for his hemoptysis. Our patient was discharged on POD 3. No further episodes of hemoptysis have occurred during 2 year-follow up. CT angiogram of the chest 1 month later demonstrated no aberrancy of pulmonary vessels or AVMs.

\section{Discussion}

Hemoptysis is a rare, albeit potentially life-threatening, condition in pediatric patients. Dual circulatory systems, bronchial and pulmonary, supply blood to the lungs. The bronchial circulation is high pressure arising from the systemic circulatory system but low volume while the pulmonary circulation is high volume but low pressure. Consequently, significant volume hemoptysis arises from the bronchial circulation (Shnayder R, Needleman JP, 2018). Massive hemoptysis has been attributed to vascular anomalies such as AVMs in recent literature but aberrant vessels have been a rarer cause (Villardo RJM et al, 2011). In adult patients, shunts involving the systemic and pulmonary circulations are typically acquired from chronic pulmonary disease states, malignancy or trauma. Congenital bronchopulmonary arterial connections are rarer and have been previously described as preferentially unilateral, right-sided and in males in the adult population (Hsieh CG et al, 2017). Bronchial artery embolization (BAE) was first described by Remy and colleagues in 1974. It has been widely used as treatment for hemoptysis in peditartic patients, including those with $\mathrm{CF}$, and has been shown to be safe and effective (Moynihan et al, 2021).

\section{Conclusion}

Hemoptysis is a serious and potentially fatal event that is thankfully rare in pediatric patients. A high clinical suspicion should be maintained when assessing a pediatric patient presenting with hemoptysis. Clinicians should not overlook congenital vascular anomalies as a cause due to their risk of catastrophic haemorrhage. BAE is a relatively safe method to treat hemoptysis and has been also demonstrated to be effective in pediatric patients, including those with CF (Moynihan et al, 2021). Clinicians should be cognizant of the multidisciplinary care required to treat pediatric patients with hemoptysis and ensure that services are readily available to guarantee optimal outcomes.

\section{References:}

Hsieh CG, Le T, Fogelfeld K, Kamangar N. Bronchial Artery Aneurysm with Associated Bronchial Artery to Pulmonary Artery Fistula: Treatment by Embolization. Journal of Clinical Imaging Science. 2017;7:2. doi:10.4103/2156-7514.199052 
Moynihan KM, Ge S, Sleeper LA, Lu M, Andren KG, Mecklosky J, Rahbar R, Fynn-Thompson F, Porras $\mathrm{D}$, Arnold J, et al. Life-Threatening Hemoptysis in a Pediatric Referral Center. Critical Care Medicine. 2021;49(3). doi:10.1097/ccm.0000000000004822

Pianosi P, Al-sadoon H. Hemoptysis in Children. Pediatrics in Review. 1996;17(10):344-348. doi:10.1542/pir.17-10-344

Shnayder R, Needleman JP. Hemoptysis. Pediatrics in Review. 2018;39(6):319-321. doi:10.1542/pir.20170157

Villardo RJ, Rosas-Salazar C, Spahr JE, Dohar JE. An unusual solitary endobronchial pulmonary arteriovenous malformation in a 10 year-old child. International Journal of Pediatric Otorhinolaryngology. 2011;75(11):1463-1467. doi:10.1016/j.ijporl.2011.08.008
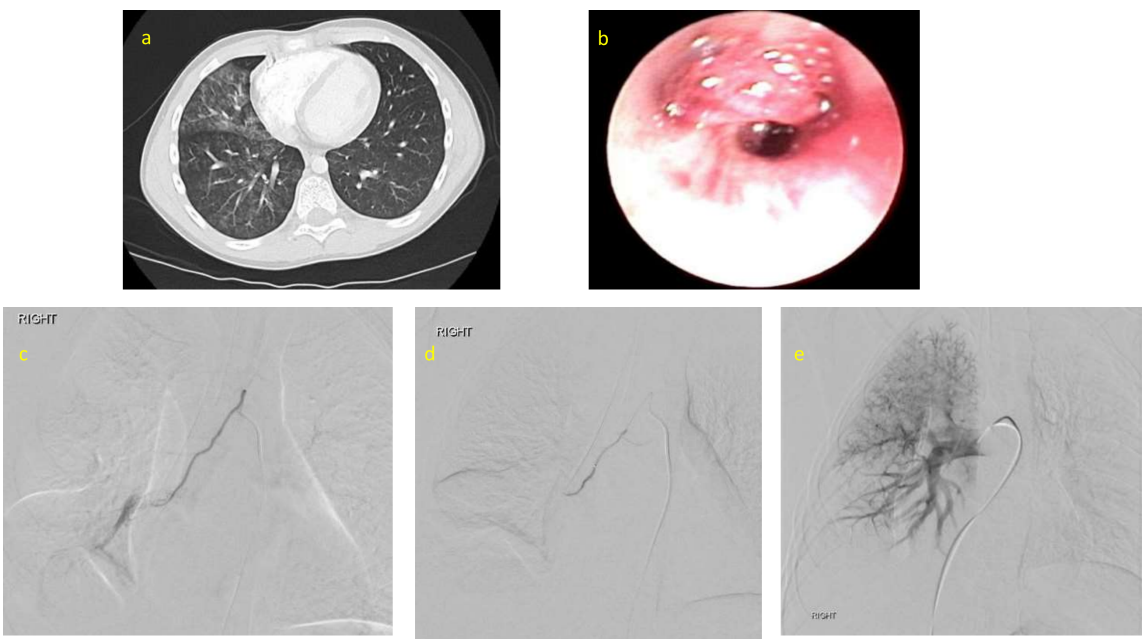